Jerusalem). Some halophytic species, such as Reaumuria hirtella excrete salt from their leaves, thus increasing the salinity of their adjacent soils; other halophytes may have the same effect as a consequence of the shedding of salt-rich leaves. The outcome is suppression of the germination and establishment of glycophytic annuals in their vicinity.

M. M. Caldwell (Utah State University, Logan) provided one example of a situation where one desert shrub had been replaced by another. The competition was between Ceratoides lanata (C3 species) and Atriplex confertifolia (C4 species) in the Great Basin Desert of North America. Carbon from the roots of Ceratoides had $\delta^{13} \mathrm{C}$ values of -22 to -25 , whereas that from Atriplex had values of -18 to -19 , which is to be expected from $\mathrm{C} 3$ and C4 plants respectively. But when soil organic matter carbon was examined for the layers beneath areas now occupied by Ceratoides it was found that upper levels had a $\delta^{13} \mathrm{C}$ of -20 to -23 , whereas deeper layers provided values of -15 to -18 . Evidently the C3 species has invaded and replaced Atriplex in areas which once it dominated. The $\mathrm{C} 4$ system does not always come out on top.

To say that competitive interactions within or between species in deserts are unimportant is thus an oversimplification of the actual state of affairs. It may be that the adaptations which competitive selection has imposed upon desert species are subtle and must be sought at a metabolic level.

\title{
Does Venus have an intrinsic magnetic field?
}

\section{from C. T. Russell}

DECEMBER on Venus promises to be very busy this year. Every 18 months the energy required to launch a probe to Venus reaches a minimum. During this year's energy minimum or, as it is more commonly called. launch opportunity, the USA and the USSR each launched two spacecraft to Venus. The two US spacecraft are scheduled to arrive on 4 December. (an orbiter) and on 9 December, (a 'bus' carrying one large and three small atmospheric probes). The USSR spacecraft are expected to arrive at Venus in late December and are generally believed to consist each of a lander with a flyby 'bus' rather than an orbiter. Thus in December. we expect that Venus will be visited by six atmospheric probes. two of which will make measurements of the Venusian surface. one bus spacecraft that will burn up in the atmosphere and two that will pass by, and an orbiter which will stay for some time making measurements to altitudes as low as $150 \mathrm{~km}$ above the surface.

One of the key questions to be addressed by the orbiter spacecraft is whether Venus possesses an intrinsic planetary field similar to those of the Earth and Mercury. Initial observations with Mariners 2 and 5 and Venera 4 suggested that Venus might not in fact have an intrinsic magnetic field. However. reexamination of some of these earlier data (Russell Geophys. Res. Lett., 3, 125. 1976) and more recent reports of the Venera

C. T. Russell is in the Institute of Geophysics and Planetary Plysics at the University of California, Los Angeles.
9 and 10 orbital magnetic measurements (Dolginov et al. Lunar Science $I X, 256,1977)$ have provided evidence in favour of an intrinsic field. The situation is far from clear. and the recent report of plasma measurements by the Venera 9 and 10 wideangle plasma devices (Verigin et al. I. geophys. Res., 83, 3721; 1978) does little to resolve this question. There is no doubt from the Venera 9 and 10 data that the obstacle deflecting the flow over the forward hemisphere of the planet is the ionosphere. The authors suggest a stagnation point, or effective obstacle height, of $500 \mathrm{~km}$ altitude. In fact it is probably even closer to the planet than this. More recent Russian radic occultation data indicate $260 \mathrm{~km}$ to be a more likely obstacle height. Thus any intrinsic planetary field plays only a minor role in deflecting the solar wind. With the solar wind penetrating this close to the planet, charge exchange of the solar wind with the hydrogen geocorona could be an important mechanism in decelerating the solar wind ahead of the planet as suggested by Wallis (Natlire, 233, 23; 1971). This mechanism would at a very minimum in the subsolar region weaken the Venus bow shock which slows down and heats the supersonic solar wind before it hits the ionosphere. The authors reject this hypothesis but show no data in the subsolar region. It is still possible that no well-defined shock transition exists in this region.

Verigin et al, also report on measurements in the wake region behind Venus. Here they find a plasma magnetic tail similar in many respects to the terrestrial magnetotail in which a "magnetic field prevents plasma from filling the region behind the obstacle." However, the authors leave open the question as to whether the tail field arises from intrinsic or ionospheric sources.

Verigin et al. also compare the Venera data with the Soviet Mars data. In many respects the Mars and Venus plasma measurements are similar, but Mars seems to stand off the solar wind further above the ionosphere than Venus. The authors suggest that this fact indicates that the Martian obstacle to the solar wind is different from the Venusian obstacle, and that the Martian obstacle is indeed an intrinsic field. On the other hand, I find the other evidence for a Martian magnetic field less convincing than for Venus (Geophys. Res. Lett., 5, $81 ; 1978)$.

In short the question of the existence of intrinsic planetary magnetic fields for the Earth's two nearest neighbors is still very controversial. We expect that further analysis of the Venera 9 and 10 data and the new data to be acquired in December will resolve this question for Venus. However. there are no plans to my knowledge to probe the Martian magnetic field until at least 1982. At that time the American Galileo spacecraft, as presently scheduled. will whiz by at an altitude of closest approach of $275 \mathrm{~km}$ on its way to Jupiter. If I were polled on the eventual outcome of these investigations I would vote yes for a presently operating Venusian magnetic dynamo and no for a Martian dynamo.

\section{Sex pheromones in bacteria}

\section{from J. R. Saunders}

TIIE role of sex pheromones in mating is well established in higher organisms and fungi. In contrast there is scant evidence for such chemical sex attractants in bacteria. In Gram-negative bacteria the formation of aggregates of bacteria during conjugation is well known (see for example, Achtman \& Skurray in Microbial Interactions (Series B. Receptors and Recognition Vol 2) 233 (ed. Reissig) Chapman and Hall, London, 1977). The molecular mechanisms involved in genetic transfer during hacterial conjugation have been intensively studied but much less is known of the signalling processes which might initiate contact 